\title{
Adherence to a Mediterranean diet and Alzheimer's disease risk in an Australian population
}

\author{
S Gardener ${ }^{1,2}, \mathrm{Y} \mathrm{Gu}^{3,4}$, SR Rainey-Smith ${ }^{1,2}$, JB Keogh ${ }^{5}, \mathrm{PM} \mathrm{Clifton}^{6,7}, \mathrm{SL}_{\text {Mathieson }}^{2}, \mathrm{~K}_{\text {Taddei }}{ }^{1,2}$, A Mondal $^{1,2}$, \\ VK Ward ${ }^{1,2}$, N Scarmeas ${ }^{3,4,8}$, M Barnes ${ }^{9}$, KA Ellis ${ }^{10,11,12}$, R Head ${ }^{9}$, CL Masters ${ }^{10,13}$, D Ames ${ }^{11,12}$, SL Macaulay ${ }^{14}$, \\ CC Rowe ${ }^{15}$, C Szoeke ${ }^{14}$ and RN Martins ${ }^{1,2}$, for the AIBL Research Group ${ }^{16}$
}

The Mediterranean diet (MeDi), due to its correlation with a low morbidity and mortality for many chronic diseases, has been widely recognised as a healthy eating model. We aimed to investigate, in a cross-sectional study, the association between adherence to a MeDi and risk for Alzheimer's disease (AD) and mild cognitive impairment (MCl) in a large, elderly, Australian cohort. Subjects in the Australian Imaging, Biomarkers and Lifestyle Study of Ageing cohort (723 healthy controls (HC), $98 \mathrm{MCl}$ and 149 AD participants) completed the Cancer Council of Victoria Food Frequency Questionnaire. Adherence to the $\mathrm{MeDi}$ (0- to 9-point scale with higher scores indicating higher adherence) was the main predictor of $\mathrm{AD}$ and $\mathrm{MCl}$ status in multinominal logistic regression models that were adjusted for cohort age, sex, country of birth, education, apolipoprotein E genotype, total caloric intake, current smoking status, body mass index, history of diabetes, hypertension, angina, heart attack and stroke. There was a significant difference in adherence to the MeDi between HC and AD subjects $(P<0.001)$, and in adherence between $\mathrm{HC}$ and $\mathrm{MCl}$ subjects $(P<0.05)$. MeDi is associated with change in Mini-Mental State Examination score over an 18-month time period $(P<0.05)$ in $\mathrm{HCs}$. We conclude that in this Australian cohort, AD and $\mathrm{MCl}$ participants had a lower adherence to the MeDi than HC participants.

Translational Psychiatry (2012) 2, e164; doi:10.1038/tp.2012.91; published online 2 October 2012

\section{Introduction}

Current literature regarding the impact of individual nutrients or food items on Alzheimer's disease (AD) risk is inconsistent, partly because humans eat meals with complex combinations of nutrients or food items that are likely to be synergistic, thereby creating complications when determining which of the dietary components are exerting a protective or adverse effect. In the case of antioxidants, for example, there is evidence in cardiovascular disease literature showing that cofactors found in whole foods are required for some antioxidants to be fully effective. Furthermore, the magnitude of effect of single antioxidants may not be sufficient for a clinical impact to be observed. ${ }^{1}$ As an alternative approach, dietary pattern analysis has emerged, one of these dietary patterns is the Mediterranean diet (MeDi).

The MeDi has received much attention for its role in the prevention of $A D$. The MeDi is characterised by a high intake of vegetables, legumes, fruits, cereals, fish and unsaturated fatty acids (mostly in the form of olive oil), low intake of saturated fatty acids, meat and poultry, low-to-moderate intake of dairy products (mostly cheese and yoghurt) and a regular but moderate amount of alcohol (mostly wine and generally with meals). This diet includes many dietary components reported to be beneficial in reducing AD risk. ${ }^{2}$ For example, many vegetables, fruits, legumes and cereals contain antioxidants, which combat oxidative stress. Previous studies in New York and Chicago have found that higher adherence to the MeDi was associated with lower AD risk. ${ }^{3,4} \mathrm{~A}$ study in France found that the MeDi may reduce the rate of cognitive decline in the elderly. ${ }^{5}$ Currently, there are no studies replicating and confirming these findings in an Australian population.

Higher adherence to a MeDi has been associated with lower risk for dyslipidemia, ${ }^{6,7}$ hypertension, ${ }^{6-9}$ abnormal glucose metabolism ${ }^{7,8}$ and coronary heart disease ${ }^{7,10-12}$ all thought to increase $A D$ risk. Furthermore, greater

\footnotetext{
${ }^{1}$ Centre of Excellence for Alzheimer's Disease Research and Care, School of Medical Sciences, Edith Cowan University, Joondalup, Western Australia, Australia; ${ }^{2}$ Sir James McCusker Alzheimer's Disease Research Unit (Hollywood Private Hospital), Perth, Western Australia, Australia; ${ }^{3}$ Taub Institute for Research of Alzheimer's Disease and the Ageing Brain, Columbia University, New York, NY, USA; ${ }^{4}$ Gertrude H. Sergievsky Centre, Columbia University, New York, NY, USA; ${ }^{5}$ School of Pharmacy and Medical Sciences and Sansom Institute for Health Research, Division of Health Sciences, University of South Australia, Adelaide, Australia; ${ }^{6}$ Department of Medicine and Department of Biomedical Science, University of Adelaide, Adelaide, Australia; ${ }^{7}$ Nutritional Interventions, Baker IDI Heart and Diabetes Institute, Melbourne, Victoria, Australia; ${ }^{8}$ Department of Neurology, Columbia University College of Physicians and Surgeons, New York, NY, USA; ${ }^{9} \mathrm{CSIRO}$, Preventative Health Flagship, Adelaide, Australia; ${ }^{10}$ Mental Health Research Institute, The University of Melbourne, Parkville, Victoria, Australia; ${ }^{11}$ National Ageing Research Institute, Parkville, Victoria, Australia; ${ }^{12}$ Department of Psychiatry, Academic Unit for Psychiatry of Old Age, The University of Melbourne, St Vincent's Aged Psychiatry Service, St George's Hospital, Victoria, Australia; ${ }^{13}$ Centre for Neuroscience, The University of Melbourne, Parkville, Victoria, Australia; ${ }^{14}$ CSIRO Preventative Health Flagship, CMSE Parkville, Parkville, Victoria, Australia and ${ }^{15}$ Department of Nuclear Medicine and Centre for PET, Austin Health, Heidelberg, Victoria, Australia

Correspondence: Professor R Martins, Centre of Excellence for Alzheimer's Disease Research and Care, School of Medical Sciences, Edith Cowan University, 270 Joondalup Drive, Joondalup, Western Australia 6027, Australia.

E-mail: r.martins@ecu.edu.au


Keywords: AlBL; Alzheimer's disease; Australia; Mediterranean diet; MCI

Received 14 June 2012; revised 16 August 2012; accepted 18 August 2012
} 
adherence to a MeDi has been associated with lower levels of cerebrovascular disease, which is also considered a risk factor for AD. ${ }^{13}$

The current cross-sectional analysis was designed to investigate the association between adherence to the MeDi and $A D$ risk in a large, elderly, Australian cohort. The results could then be compared with previous findings in the North American and French populations.

\section{Materials and methods}

Samples and procedures. The Australian Imaging, Biomarkers and Lifestyle Study of Ageing (AIBL) study ${ }^{14}$ is a longitudinal study of 1112 volunteers including healthy control $(\mathrm{HC})$, mild cognitive impairment $(\mathrm{MCl})$ and $A D$ individuals who are being assessed for prospective research into ageing and AD. The cohort comprised $768 \mathrm{HC}, 133 \mathrm{MCl}$ and $211 \mathrm{AD}$ individuals at baseline. All AIBL volunteers were aged 60 years and above at baseline, and excluded if they had a history of non-AD dementia, schizophrenia, bipolar disorder, significant current depression, Parkinson's disease, cancer (other than basal cell skin carcinoma) within the last 2 years, symptomatic stroke, insulin-dependent diabetes, uncontrolled diabetes mellitus or current regular alcohol use exceeding two standard drinks per day for women or four per day for men. During clinical review panels, a consensus diagnosis was assigned for each participant, which included consideration of diagnostic criteria (DSM-IV (Diagnostic and Statistical Manual of Mental Disorders, Fourth Edition) diagnosis $^{15}$ and International Classification of Disease (ICD)-10 diagnosis ${ }^{16}$ ). Where appropriate, ICD-10 dementia severity rating, ${ }^{16}$ NINCDS-ADRDA AD diagnosis (probable or possible) and $\mathrm{MCl}$ classifications were applied. The clinical review panel comprised old age psychiatrists, a neurologist, a geriatrician and neuropsychologists. $\mathrm{MCl}$ diagnoses were made according to the protocol based upon the criteria of Winblad et al., ${ }^{17}$ which are informed by the criteria of Petersen et al. ${ }^{18}$ Consistent with Winblad criteria, all participants classified with $\mathrm{MCl}$ had either personally, or through an informant, reported memory difficulties. ${ }^{17}$ Subjects were given written instructions of the risks and benefits of their participation in the study, and signed informed consent was obtained before data collection commenced. The AIBL study was approved by the Institutional Ethics Committees of the Austin Health, St Vincent's Health, Hollywood Private Hospital and Edith Cowan University. ${ }^{14}$

18-Month follow-up assessment. Of the $723 \mathrm{HCs}$ who completed the Cancer Council of Victoria Food Frequency Questionnaire (CCVFFQ) at baseline, 664 returned for followup assessment after 18 months. The remaining $59 \mathrm{HC}$ participants either withdrew from the study between visits, or were deceased at the time of their follow-up appointment. In all, 12 participants were excluded from the analysis due to incomplete neuropsychological test results, leaving a total of $652 \mathrm{HCs}$ in the analysis. The follow-up assessment included a neuropsychological test battery, which had also been completed at baseline assessment, enabling generation of repeated cognitive performance data. For the purpose of the analysis, the results from the following four neuropsychological tests were used; the Mini-Mental State Examination, Logical Memory II (WMS; Story 1 only), California Verbal Learning Test II-Second Edition (long delay) and Delis-Kaplan Executive Function System Verbal Fluency.

Genotyping. Fasting blood samples were obtained using standard venipuncture of the antecubital vein and collected into EDTA tubes containing prostaglandin E1 (Sapphire Biosciences, Sydney, NSW, Australia, $33 \cdot 3 \mathrm{ng} \mathrm{ml}^{-1}$ ) to prevent platelet activation. Whole blood was stored at $-80^{\circ} \mathrm{C}$ in $200 \mu \mathrm{l}$ aliquots, thawed then centrifuged to separate leucocytes when required. DNA was isolated from the leucocytes, using the Qiagen Midiprep kits (Hilden, Germany), and apolipoprotein E (APOE) genotype was determined using PCR amplification and restriction enzyme digest techniques. ${ }^{19}$

\section{Evaluation}

Diet. Of the baseline cohort, $723 \mathrm{HC}, 98 \mathrm{MCl}$ and $149 \mathrm{AD}$ subjects completed the CCVFFQ. The 74-item self-administered semi-quantitative FFQ assesses usual daily food intake over the preceding 12 months. The CCVFFQ has previously been validated relative to 7 -day weighed food records. ${ }^{20}$ The responses were analysed by the Cancer Council in Carlton, Victoria, Australia. The food composition data used to calculate daily nutrient intake originates from the Nutrient Tables for use in Australia 1995 (NUTTAB95).

The HCs in the AIBL cohort completed the CCVFFQ themselves. The $\mathrm{MCl}$ subjects had cognitive dysfunction, which did not significantly restrict the performance of activities of daily living; therefore they were also able to complete the CCVFFQ without requiring assistance. The majority of $A D$ participants in the AIBL study who completed the CCVFFQ at baseline were early stage, mild AD; most were living at home with carers, normally a spouse or family member. The AD participant completed the CCVFFQ with assistance from the carer; alternatively, the carer validated the completed questionnaire afterwards. In each case, the carer lived with the AD participant, so either prepared the meals for them or was aware of meals they consumed.

We followed the most commonly described method to construct the MeDi score. ${ }^{10}$ An individual was assigned a value of 1 for each; beneficial component (fruits, vegetables, legumes, cereals and fish) if his/her caloric-adjusted consumption was at or above the gender-specific median; for each detrimental component (meat and dairy products) where caloric-adjusted consumption was below the gender-specific median; and for a ratio of monounsaturated fats to saturated fats at or above the median. Individuals were also assigned a value of 1 for mild to moderate alcohol consumption ( $>5$ to $<25 \mathrm{~g}$ per day for females and $>10$ to $<50 \mathrm{~g}$ per day for males). The MeDi score was generated for each participant by adding the scores for each category. Thus, the MeDi score could range from 0 to 9 , with a higher score indicating a greater adherence to the MeDi. ${ }^{21}$

Covariates. Analyses were adjusted for age (years), education ( $\leqslant 12$ and $>12$ years), current smoking status, body mass index (BMI; calculated as weight in kilograms divided by height in metres squared) and total energy intake (kcal). 
Country of birth was used as a dichotomous variable (Australian or other). APOE genotype was also used dichotomously: the absence of $\varepsilon 4$ allele vs presence of either one or two $\varepsilon 4$ alleles. A history of diabetes, hypertension, angina, heart attack and stroke were defined by self-report.

Missing variable data. The only variable with missing data was BMI. We used multivariate imputation by chain equation to provide BMI values for 102 participants. It was observed that the distribution of the imputed BMI data was comparable to that of the initial BMI data, thus we deemed the imputed $\mathrm{BMI}$ values to be acceptable for inclusion in the subsequent analysis.

Statistical analyses. All statistical analyses were conducted using the R Statistical Software (version 2.12.2; the $\mathrm{R}$ Foundation for Statistical Computing, Vienna, Austria), except the 18-month follow-up assessment analysis, which was conducted using the statistical package for the social sciences (SPSS, Chicago, IL, USA) version 19.

Multinomial logistic regression analyses. Data were distributed across three groups, $\mathrm{HC}, \mathrm{MCl}$ and $\mathrm{AD}$. All categorical predictors were used as factors. $\mathrm{HC}$ was the reference category.

Basic model. We calculated multinomial logistic regression models with diagnosis at baseline evaluation as the outcome: $\mathrm{HC}$ vs $\mathrm{AD}$ or $\mathrm{MCl}$. The main predictor was the baseline visit MeDi score as an ordinal score.

Adjusted model. Owing to demographic and clinical differences between the three classification groups, in subsequent models we simultaneously adjusted for the following: age at assessment, sex, country of birth, education, APOE $\varepsilon 4$ allele status, current smoking status and BMI. Although caloric intake adjusted residuals were used in the MeDi score calculation, we also included caloric intake as a covariate in the models (as recommended by Willet and Stampfer ${ }^{22}$ ).

Fully adjusted model with vascular comorbidities. We simultaneously included all vascular risk factors (history of stroke, diabetes, hypertension, angina and heart attack) in the fully adjusted logistic regression model.

Using raw scores from the neuropsychological tests, a $\lambda$ score was generated for each participant indicating change in each neuropsychological test result between the baseline and 18-month follow-up assessment. A partial correlation was performed individually for each set of $\lambda$ scores for each of the four neuropsychological tests, adjusted for the covariates listed previously.

\section{Results}

Compared with $\mathrm{HC}$ subjects, subjects with $\mathrm{AD}$ had a lower mean MeDi score $(P<0.001)$; and compared with $\mathrm{HC}$ subjects, subjects with $\mathrm{MCl}$ had a lower mean MeDi score $(P<0.05$; Table 1 and Figure 1$) . \mathrm{MCl}$ compared with $\mathrm{AD}$ lacks statistical significance.

Higher adherence to the MeDi was seen in HCs relative to $A D$ subjects. The results were similar in both adjusted and unadjusted models (Table 2). The $P$-values, as shown in Table 2, indicate that the overall effect of the MeDi is statistically significant.

Compared with the reference $\mathrm{HC}$ category, each additional unit in the MeDi score was associated with 13-19\% lower

Table 1 Demographic, clinical and dietary characteristics for subjects with $\mathrm{AD}, \mathrm{MCl}$ and $\mathrm{HC}$

\begin{tabular}{|c|c|c|c|c|c|}
\hline Characteristics & $A / /(\mathrm{n}=970)$ & $A D(\mathrm{n}=149)$ & $M C l(\mathrm{n}=98)$ & $H C(\mathrm{n}=723)$ & P-value ${ }^{a}$ \\
\hline $\begin{array}{l}\text { Age, years; mean (s.d.) } \\
\text { Gender, men; no. (\%) } \\
\text { Education } \leq 12 \text { years; no. (\%) } \\
\text { MMSE; mean (s.d.) } \\
\text { Presence of APOE } \& 4 \text { allele; no. (\%) } \\
\text { Energy intake, Kcal; mean (s.d.) } \\
\text { BMI; mean (s.d.) }\end{array}$ & $\begin{aligned} & 71.72(7.86) \\
& 407(42) \\
& 477(49) \\
& 27.07(4.34) \\
& 331(34) \\
& 1695(599) \\
& 26.16(4.23)\end{aligned}$ & $\begin{aligned} & 77.50(8.20) \\
& 63(42) \\
& 89(60) \\
& 19.10(5.22) \\
& 93(62) \\
& 1702(616) \\
& 24.80(4.34)\end{aligned}$ & $\begin{aligned} & 76.01(7.78) \\
& 41(42) \\
& 53(54) \\
& 26.16(2.70) \\
& 52(53) \\
& 1710(618) \\
& 25.56(3.75)\end{aligned}$ & $\begin{array}{r}69.94(6.95) \\
303(42) \\
335(46) \\
28.86(1.19) \\
186(26) \\
1691(594) \\
26.45(4.22)\end{array}$ & $\begin{array}{l}0.000 \\
0.996 \\
0.007 \\
0.000 \\
0.000 \\
0.942 \\
0.000\end{array}$ \\
\hline $\begin{array}{l}\text { Smoking; no. (\%) } \\
\quad \text { Past } \\
\quad \text { Current } \\
\text { Country of birth, Australian; no. (\%) } \\
\text { Diabetes; no. (\%) } \\
\text { Hypertension; no. (\%) } \\
\text { Angina; no. (\%) } \\
\text { Heart attack; no. (\%) } \\
\text { Stroke; no. (\%) } \\
\text { MeDi; mean (s.d.) }\end{array}$ & $\begin{aligned} & 444(46) \\
& 29(3) \\
& 687(71) \\
& 76(8) \\
& 387(40) \\
& 70(7) \\
& 50(5) \\
& 33(3) \\
& 4.40(1.64)\end{aligned}$ & $\begin{array}{c}74(50) \\
0(0) \\
105(70) \\
14(9) \\
56(38) \\
14(9) \\
10(7) \\
17(11) \\
3.79(1.57)\end{array}$ & $\begin{array}{c}51(52) \\
4(4) \\
63(64) \\
11(11) \\
39(40) \\
13(13) \\
7(7) \\
2(2) \\
4.02(1.63)\end{array}$ & $\begin{aligned} & 319(44) \\
& 25(3) \\
& 519(72) \\
& 51(7) \\
& 292(40) \\
& 43(6) \\
& 33(5) \\
& 14(2) \\
& 4.57(1.62)\end{aligned}$ & $\begin{array}{l}0.197 \\
0.063 \\
0.308 \\
0.263 \\
0.817 \\
\mathbf{0 . 0 1 7} \\
0.360 \\
\mathbf{0 . 0 0 0} \\
\mathbf{0 . 0 0 0}\end{array}$ \\
\hline $\begin{array}{l}\text { MeDi tertiles; no. (\%) } \\
\text { Low }(0-3) \\
\text { Middle }(4-5) \\
\text { High }(6-9)\end{array}$ & $\begin{array}{l}289(30) \\
428(44) \\
253(26)\end{array}$ & $\begin{array}{l}66(44) \\
65(44) \\
18(12)\end{array}$ & $\begin{array}{l}36(37) \\
44(45) \\
18(18)\end{array}$ & $\begin{array}{l}187(26) \\
319(44) \\
217(30)\end{array}$ & 0.000 \\
\hline
\end{tabular}

Abbreviations: AD, Alzheimer's disease; APOE, apolipoprotein E; BMI, body mass index; HC, healthy control; $\mathrm{MCl}$, mild cognitive impairment; MeDi, Mediterranean diet; MMSE, Mini-Mental State Examination.

${ }^{a}$ Bold values indicate statistical significance $(P<0.05)$; characteristics compared using analysis of variance for continuous variables and $\chi^{2}$ for categorical variables. ${ }^{b} \mathrm{BMI}$ is calculated as weight in kilograms divided by height in metres squared. 'History of diabetes, hypertension, angina, heart attack or stroke. 


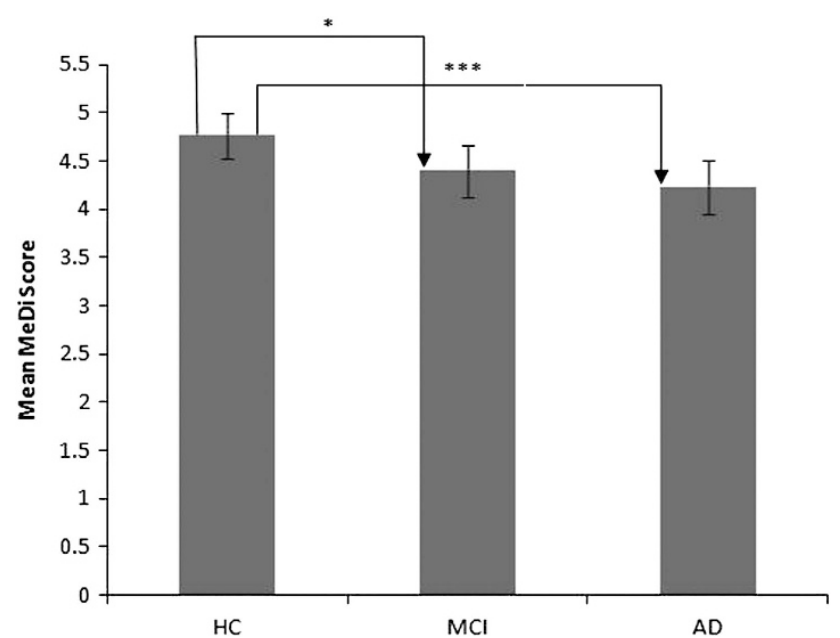

Figure 1 Comparison of mean MeDi score using general linear model adjusted for necessary covariates. Bars represent mean ( \pm s.e.); general linear model adjusted for necessary covariates; ${ }^{\star} P<0.05,{ }^{* \star \star} P<0.001$. Adjusted for age, sex, education, apolipoprotein $\mathrm{E}$ genotype, country of birth (Australia vs other), body mass index, total caloric intake, current smoking status and history of hypertension, angina, stroke, diabetes and heart attack. Abbreviations: AD, Alzheimer's disease; $\mathrm{HC}$, healthy control; $\mathrm{MeDi}$, Mediterranean diet; $\mathrm{MCl}$, mild cognitive impairment.

odds of being in the $\mathrm{MCl}$ category, and $19-26 \%$ lower odds of being in the AD category.

A significant correlation $(P<0.05)$ was observed between baseline MeDi score and change in Mini-Mental State Examination score between baseline and 18-month follow-up assessment in $\mathrm{HC}$ participants. No significant correlation was observed between baseline MeDi score and the other three neuropsychological test scores in healthy individuals (Table 3). Supplementary data shows demographic and clinical characteristics by MeDi score tertile in all clinical classification groups (Supplementary Table 1), and in HC group alone (Supplementary Table 2).

\section{Discussion}

Our analysis suggests that lower adherence to the MeDi is associated with $\mathrm{MCl}$ and $\mathrm{AD}$. This is consistent with previous studies. ${ }^{3-5}$ This is the first study on AD to use an elderly Australian cohort to replicate findings described in US and French populations. Australia is a geographically isolated country with a different ethnic mix and it is interesting that our results concur with those previously described. The US population was taken from the Northern Manhattan area; it comprised 2258 non-demented individuals who were assessed at intervals of $\sim 18$ months. These participants had a mean age of 77.2 with a s.d. of 6.6 at baseline assessment. Over the course of 4 years there were 262 incident $A D$ cases, and the investigators concluded that higher adherence to the MeDi was associated with lower $A D$ risk. The French study cohort comprised 1410 individuals aged 65 and over; all were non-demented at baseline and underwent a follow-up assessment within 5 years. Cognitive performance was assessed using the outcome of four neuropsychological tests, including Mini-Mental State Examination; the investigators concluded that each additional unit of
Table 2 Odds ratio of being classified as $\mathrm{AD}$ or $\mathrm{MCl}$ compared with a healthy control classification

\begin{tabular}{|c|c|c|c|c|c|}
\hline Model $^{\mathrm{a}}$ & $\begin{array}{l}\text { Number of } \\
\text { subjects } \\
\text { included in } \\
\text { model }\end{array}$ & Classification & OR & $95 \% \mathrm{Cl}$ & $\begin{array}{c}\mathrm{P} \text { - } \\
\text { value }\end{array}$ \\
\hline \multirow[t]{3}{*}{ Model 1} & 970 & $\mathrm{HC}$ & $\begin{array}{c}1.00 \\
\text { (reference) }\end{array}$ & & \\
\hline & & $\mathrm{MCl}$ & 0.809 & $0.71-0.92$ & $\star *$ \\
\hline & & $A D$ & 0.740 & $0.66-0.83$ & $\star * \star$ \\
\hline \multirow[t]{3}{*}{ Model 2} & 970 & $\mathrm{HC}$ & $\begin{array}{c}1.00 \\
\text { (reference) }\end{array}$ & & \\
\hline & & $\mathrm{MCl}$ & 0.863 & $0.75-1.00$ & * \\
\hline & & AD & 0.813 & $0.71-0.93$ & $\star \star$ \\
\hline \multirow[t]{3}{*}{ Model 3} & 970 & $\mathrm{HC}$ & $\begin{array}{c}1.00 \\
\text { (reference) }\end{array}$ & & \\
\hline & & $\mathrm{MCl}$ & 0.866 & $0.75-1.00$ & * \\
\hline & & $A D$ & 0.806 & $0.71-0.92$ & $\star *$ \\
\hline
\end{tabular}

Abbreviations: AD, Alzheimer's disease; APOE, apolipoprotein E; Cl, confidence interval; $\mathrm{HC}$, healthy control; $\mathrm{MCl}$, mild cognitive impairment; OR, odds ratio.

Multinomial logistic regression analysis; ${ }^{\star} P<0.05$, ${ }^{* \star} P<0.01$ and ${ }^{* \star *} P<0.001$. aModel 1: unadjusted. Model 2: adjusted for age at assessment, sex, country of birth, education, APOE E4 allele status, current smoking status, caloric intake and BMI. Model 3: adjusted for covariates in model 2 and history of stroke, diabetes, hypertension, angina and heart attack.

Table 3 Partial correlation results showing baseline MeDi score is correlated with change in cognitive performance in $\mathrm{HC}$

\begin{tabular}{lcr}
\hline$\lambda$ For neuropsychological test & Significance $^{a}$ & Correlation $^{-}$ \\
\hline MMSE & $\mathbf{0 . 0 1 4}$ & 0.098 \\
LM II & 0.779 & -0.011 \\
D-KEFS Verbal Fluency & 0.294 & 0.042 \\
CVLT II Long Delay & 0.472 & 0.029
\end{tabular}

Abbreviations: CVLT II, California Verbal Learning Test-Second Edition; D-KEFS, Delis-Kaplan Executive Function System; HC, healthy control; LM II Logical Memory II (WMS; Story 1 only); MeDi, Mediterranean diet; MMSE, Mini-Mental State Examination.

${ }^{a}$ Bold value indicates statistical significance $(P<0.05)$; partial correlation.

MeDi score was associated with fewer Mini-Mental State Examination errors at follow-up.

The associations between MeDi and AD reported in this study remained unchanged and significant even when adjusted for the most commonly considered potential confounders for $A D$, such as age, sex, education, APOE genotype, country of birth (Australia vs other), caloric intake, $\mathrm{BMI}$, current smoking status, hypertension, angina, stroke, diabetes and heart attack.

The only other Australian study to investigate the association between MeDi and cognitive decline was the PATH through life study conducted in Canberra. ${ }^{23}$ This cohort comprised 1528 cognitively normal individuals aged 60-64 years. Individuals were tested at two time points, 4 years apart. After the follow-up assessment, 10 participants were diagnosed with $\mathrm{MCl}, 10$ participants with a clinical dementia rating of 0.5 and 37 participants presented with any mild cognitive disorder. The authors observed that greater adherence to the MeDi was not protective against cognitive impairment, though they did find that diet shares important associations with cognitive health. The apparent lack of protection afforded by the MeDi may be explained by the heterogeneous nature of the study population. Furthermore, 
only 66 participants from the original 1528 underwent any cognitive change in the 4 years between assessments; this low number may have resulted in insufficient statistical power to detect MeDi effects.

Most studies have excluded $\mathrm{MCl}$ individuals from their investigations, resulting in a lack of information on factors that may promote conversion to AD. However, consistent with our findings, a study by Scarmeas et al. ${ }^{24}$ found a higher adherence to the MeDi is associated with a trend of reduced risk of developing $\mathrm{MCl}$, and reduced risk of $\mathrm{MCl}$ conversion to AD. Another study from the United States comprising 1640 cognitively normal and $329 \mathrm{MCl}$ subjects also found a higher MeDi score may be beneficial to cognitive function, and observed a borderline reduced risk of $\mathrm{MCl}$ incidence and conversion to dementia. ${ }^{25}$

One of the possible mechanisms through which the MeDi may be exerting its protective effect for AD could be via effects on the vascular system. ${ }^{26-28}$ There is strong evidence linking the MeDi to lower incidence of vascular disease-associated conditions such as dyslipidemia, ${ }^{6,7}$ hypertension, ${ }^{6-9}$ abnormal glucose metabolism ${ }^{7,8}$ and coronary heart disease, ${ }^{7,10-12}$ which are also risk factors for AD. Scarmeas et al. ${ }^{29}$ investigated whether there was attenuation of the association between MeDi and $A D$ when vascular variables (stroke, diabetes mellitus, hypertension, heart disease and lipid levels) were simultaneously introduced in their logistic regression models, which used diagnosis at baseline evaluation as the dichotomous outcome and MeDi score as the main predictor. They noted that their association with the MeDi and AD is not mediated by vascular comorbidities, concluding it could be the result of other biological pathways or measurement error in the vascular variables. Scarmeas et $a .^{13}$ also demonstrated an association between the MeDi and cerebrovascular disease, specifically magnetic resonance imaging infarcts: subjects in the highest tertile of MeDi adherence had a reduction in odds for brain infarcts of about $40 \%$. This study also found that among cardiovascular risk factors, only hypertension was associated with brain infarcts in adjusted models.

Oxidative stress could be another biological mechanism linking the MeDi and $A D$. AD brains exhibit constant evidence of reactive oxygen species and reactive nitrogen speciesmediated injury. These reactive species, formed during normal metabolic processes, are generally unstable and highly reactive; for this reason their levels are normally kept low by efficient antioxidant systems. In some circumstances, their production can exceed the antioxidant ability to destroy them, and oxidative stress occurs. ${ }^{30}$ Increasing the intake of antioxidants should theoretically counteract the detrimental effects of oxidative stress and thereby reduce $A D$ risk. Complex phenols and many other substances with important antioxidant properties, such as olive oil, ${ }^{31}$ wine, fruits and vegetables, vitamins $\mathrm{C}, \mathrm{E}, \mathrm{B} 12$, folate and carotenoids, ${ }^{32-37}$ are found in high concentrations in the typical components of the MeDi, ${ }^{38}$ and may reduce markers of oxidative stress. The MeDi could be capturing the composite influence of dietary antioxidants and this could, at least partially, provide an explanation for the observed association with a lower risk of AD.

The protective role of the MeDi against cognitive decline may also be mediated by attenuation of the inflammation pathway. Inflammation has been found to be associated with a higher risk for $A D$ and cognitive decline. Higher adherence to the MeDi has been associated with lower $\mathrm{C}$-reactive protein levels (an inflammatory marker that has been detected in neuritic plaques and neurofibrillary tangles in the brains of patients with $A D$ and is upregulated in $A D$ brains and serum), in both observational ${ }^{6,39,40}$ and interventional studies. ${ }^{8,41}$ However, a prospective study by $\mathrm{Gu}$ et al. $^{21}$ concluded that the favourable association between better adherence to the MeDi and lower risk of $A D$ did not seem to be mediated by high-sensitivity C-reactive protein, fasting insulin or adiponectin (an adipokine, with low levels linked to metabolic syndrome and inflammation). High-sensitivity C-reactive protein was used as an index of systemic inflammation, and fasting insulin and adiponectin as indices of metabolic profile. The authors suggested that other aspects of inflammatory and metabolic pathways not captured by these biomarkers may be relevant to the MeDi-AD association, for example, interleukin- 6 . $^{21}$

Our study was conducted in an Australian cohort, which is unlikely to adhere strictly to a diet typical of Mediterranean countries. Therefore, 'true MeDi' adherence in our population may be significantly lower when compared with Mediterranean populations, and subjects with high MeDi adherence in Australia may be potentially categorised as low MeDi adherence subjects compared with Mediterranean populations. However, our results support the notion that the beneficial effects of the MeDi are transferable to different populations.

There are limitations to our report; this is a cross-sectional study, so we cannot assume that our results show decreased adherence to the MeDi is a risk factor for $A D$, only that in our cohort the AD participants had a decreased adherence to the MeDi at baseline. This could be explained by a change in dietary patterns owing to $A D$ diagnosis. In addition, we recognise that other factors may counteract the beneficial effects of the MeDi, for example, participants with a MeDi score of 8 who nevertheless develop AD could be explained by their current smoking status, APOE genotype or other factors. It should be noted that the vast majority of our cohort are Caucasians and inferences regarding similar effects in other populations may therefore be limited.

Within the AIBL study we do not collect data pertaining to family origin, although we acknowledge the influence family origin will have on the participant's current dietary habits. It has long been recognised that cultural factors are dominant in food selection. Cultural influences lead to differences in the habitual consumption of certain foods and in traditions of preparation, and in certain cases can lead to restrictions such as exclusion of meat and milk from the diet. ${ }^{42}$ There is a dynamic influence of culture on many aspects of family lifestyle across generations. The daily practices of eating are an accumulation of exposures-not just familial and intergenerational but also social and environmental. ${ }^{43}$ Adult dietary habits are determined by familial and cultural factors rooted in childhood and adolescence, ${ }^{44}$ and family members show similarities in food preferences. ${ }^{45}$ In a study by Green et al. ${ }^{43}$ there was evidence of influences on eating across the span of three generations. Not only did culture influence what was included as part of daily life but also what was prohibited. A range of dietary restrictions were reported to varying degrees. Where restrictions such as vegetarianism or the prohibition of pork were imposed, the 
restrictions were readily accepted and were attributed to family or social tradition or religion. Fasting was perceived as a way of retaining the traditions of a culture and setting examples for children to follow. Patterns of global migration mean that large numbers of people have moved to western nations, influencing the dietary habits and lifestyle of many migrants. There is a wider variation in diet as a result of this migration. With dietary habits and lifestyle undergoing transition as a result of migration, cultural background is now relevant to many diseases. ${ }^{43}$ However, a number of studies have shown that where individuals have migrated from one country to another, they have developed conditions commonly seen in the country they emigrate to, for example, increased incidence of cardiovascular disease and cancer in US residents born overseas. ${ }^{46}$

The FFQ utilised in this study relied on participants' estimations of food intake over the previous 12-month period; this is a common limitation of studies of diet and disease and can lead to misclassification of exposure due to limited accuracy, and therefore misclassification of MeDi adherence. This is particularly important where the AD participants are concerned; we are relying on the carers for validation or assistance with completion of the CCVFFQ, and therefore assuming the carers are cognitively intact themselves, which may not be the case due to a large proportion of the carers being elderly spouses. Misclassification of diet is also not equal between persons with and without cognitive impairment, there is higher misclassification in $\mathrm{MCls}$ than $\mathrm{HCs}$, and this raises the potential for 'differential' misclassification bias. One option to address this limitation, which we will utilise in our subsequent longitudinal analysis, is to follow $\mathrm{HCs}$ that convert to $\mathrm{MCl}$ or $\mathrm{AD}$ between baseline and subsequent follow-ups. However, by excluding the ADs and MCls in the cohort and analysing change in neuropsychological test scores over an 18-month period, we show that diet is not confounded by cognitive performance. Based on these analyses it seems more likely that we can rely on the CCVFFQ data for MCls and $\mathrm{ADs}$ and that dietary change due to an $\mathrm{AD}$ or $\mathrm{MCl}$ diagnosis is not an issue.

The CCVFFQ is known to under-report certain foods, including soft drinks and snack foods. Moreover, the differences in the FFQ completion protocol for each cognitively intact, $\mathrm{MCl}$ and $\mathrm{AD}$ participant leads to even more 'noise' in the diet data, and we recognise that ideally a nutrient biomarker pattern that reflects the MeDi diet would be used as an approach for circumventing the limitations of using the FFQ in $\mathrm{MCl}$ and $\mathrm{AD}$ individuals. The MeDi score itself also has limitations; it weighs equally the underlying individual food categories, which in turn are composed of different numbers of food constituents. Frequencies of food intake are based on relatively few diet constituents, which may underestimate the overall quantity of food in each food category.

Despite the use of standard criteria, the diagnostic expertise of the AIBL group and the thorough workup, there is always the possibility of disease misclassification. It is also possible that diet is related to socioeconomic status or to other habits or characteristics related to better health and a lower risk for AD. We addressed this by adjusting for country of birth (Australia vs other), years of education, current smoking status, BMI, history of hypertension, angina, stroke, diabetes and heart attack. Another confounder usually considered in nutritional epidemiology is caloric intake. We used caloric intake adjusted residuals for MeDi score calculation and also included caloric intake as a covariate (as Willet and Stampfer recommended). ${ }^{22}$ However, we found that $\mathrm{ADs}$ and MCls had a lower adherence to the MeDi even after analysis with caloric intake as a covariate.

Many additional aspects of our study provide confidence in our findings. The dietary data were collected using an instrument previously validated in earlier epidemiological studies. We used an a priori developed dietary pattern. ${ }^{10}$ Measures for multiple potential risk factors have been recorded carefully and adjusted for in the analyses.

In summary, the findings of our study are similar to those reported in French and US populations. In the Australian AIBL cohort, both $\mathrm{MCl}$ and $\mathrm{AD}$ patients have a lower adherence to the MeDi compared with HCs. We intend to investigate the association between MeDi and conversion rates from $\mathrm{HC}$ to $\mathrm{MCl}$ and $\mathrm{AD}$ in longitudinal analyses.

\section{Conflict of interest}

The authors declare no conflict of interest.

Acknowledgements. Samantha Gardener is supported by a University Postgraduate Award from the Dementia Collaborative Research Centres programme. Nikolaos Scarmeas is supported by a grant from the National Institute on Ageing, R01 AG028506. Funding for the AIBL study is provided by the CSIRO Flagship Collaboration Fund and the Science and Industry Endowment Fund in partnership with Edith Cowan University, Mental Health Research Institute, Alzheimer's Australia, National Ageing Research Institute, Austin Health, CogState, Hollywood Private Hospital, Sir Charles Gardner Hospital. The study also receives funding from the National Health and Medical Research Council, the Dementia Collaborative Research Centres program, The McCusker Alzheimer's Research Foundation and Operational Infrastructure Support from the Government of Victoria. The AIBL team thank the participants in the AIBL study for their commitment and dedication to helping advance research into the early detection and causation of $A D$ and the clinicians who referred patients to the study.

Disclaimer. This study is industry sponsored. No funding source had a role in the preparation of this article or the decision to submit the paper for publication.

1. Mozaffarian D, Appel LJ, Van Horn L. Components of a cardioprotective diet: new insights. Circulation 2011; 123: 2870-2891.

2. Scarmeas N, Luchsinger JA, Mayeux R, Stern Y. Mediterranean diet and Alzheimer disease mortality. Neurology 2007; 69: 1084-1093.

3. Scarmeas N, Stern Y, Tang MX, Mayeux R, Luchsinger JA. Mediterranean diet and risk for Alzheimer's disease. Ann Neurol 2006; 59: 912-921.

4. Feart C, Samieri C, Rondeau V, Amieva H, Portet F, Dartigues JF et al. Adherence to a Mediterranean diet, cognitive decline, and risk of dementia. JAMA 2009; 302: 638-648.

5. Tangney CC, Kwasny MJ, Li H, Wilson RS, Evans DA, Morris MC. Adherence to a Mediterranean-type dietary pattern and cognitive decline in a community population. Am J Clin Nutr 2011; 93: 601-607.

6. Chrysohoou C, Panagiotakos DB, Pitsavos C, Das UN, Stefanadis C. Adherence to the Mediterranean diet attenuates inflammation and coagulation process in healthy adults: The ATTICA Study. J Am Coll Cardiol 2004; 44: 152-158.

7. Singh RB, Dubnov G, Niaz MA, Ghosh S, Singh R, Rastogi SS et al. Effect of an IndoMediterranean diet on progression of coronary artery disease in high risk patients (IndoMediterranean Diet Heart Study): a randomised single-blind trial. Lancet 2002; 360: 1455-1461.

8. Esposito K, Marfella R, Ciotola M, Di Palo C, Giugliano F, Giugliano G et al. Effect of a mediterranean-style diet on endothelial dysfunction and markers of vascular inflammation in the metabolic syndrome: a randomized trial. JAMA 2004; 292: 1440-1446.

9. Psaltopoulou T, Naska A, Orfanos P, Trichopoulos D, Mountokalakis T, Trichopoulou A. Olive oil, the Mediterranean diet, and arterial blood pressure: the Greek European Prospective Investigation into Cancer and Nutrition (EPIC) study. Am J Clin Nutr 2004; 80: 1012-1018.

10. Trichopoulou A, Costacou T, Bamia C, Trichopoulos D. Adherence to a Mediterranean diet and survival in a Greek population. N Engl J Med 2003; 348: 2599-2608.

11. de Lorgeril M, Salen P, Martin JL, Monjaud I, Delaye J, Mamelle N. Mediterranean diet, traditional risk factors, and the rate of cardiovascular complications after myocardial infarction: final report of the Lyon Diet Heart Study. Circulation 1999; 99: 779-785. 
12. Knoops KT, de Groot LC, Kromhout D, Perrin AE, Moreiras-Varela O, Menotti A et al. Mediterranean diet, lifestyle factors, and 10-year mortality in elderly European men and women: the HALE project. JAMA 2004; 292: 1433-1439.

13. Scarmeas N, Luchsinger JA, Stern Y, Gu Y, He J, Decarli C et al. Mediterranean diet and magnetic resonance imaging-assessed cerebrovascular disease. Ann Neurol 2011; 69 257-268.

14. Ellis KA, Bush Al, Darby D, De Fazio D, Foster J, Hudson P et al. The Australian Imaging Biomarkers and Lifestyle (AIBL) study of aging: methodology and baseline characteristics of 1112 individuals recruited for a longitudinal study of Alzheimer's disease. Int Psychogeriatr 2009; 21: 672-687.

15. Association AP Diagnostic and Statistical Manual of Mental Disorders. 4th Edition (ed. American Psychiatric Association: Washington, DC, 1994.

16. Organisation WHThe ICD-10 Classification of Mental and Behavioural Disorders Clinical Descriptions and Diagnostic Guidelines. World Health Organisation: Geneva, 1992

17. Winblad B, Palmer K, Kivipelto M, Jelic V, Fratiglioni L, Wahlund LO et al. Mild cognitive impairment-beyond controversies, towards a consensus: report of the International Working Group on Mild Cognitive Impairment. J Intern Med 2004; 256: 240-246.

18. Petersen RC, Smith GE, Waring SC, Ivnik RJ, Tangalos EG, Kokmen E. Mild cognitive impairment: clinical characterization and outcome. Arch Neurol 1999; 56: 303-308.

19. Hixson JE, Vernier DT. Restriction isotyping of human apolipoprotein $E$ by gene amplification and cleavage with Hhal. J Lipid Res 1990; 31: 545-548.

20. Ireland PJ, Giles D, O'Dea G, Powles K, Rutishauser J, Wahlqvist I et al. Development of the Melbourne FFQ: a food frequency questionnaire for use in an Australian prospective study involving an ethnically diverse cohort. Asia Pac J Clin Nutr 1994; 3: 19-31.

21. Gu Y, Luchsinger JA, Stern Y, Scarmeas N. Mediterranean diet, inflammatory and metabolic biomarkers, and risk of Alzheimer's disease. J Alzheimers Dis 2010; 22: 483-492.

22. Willett W, Stampfer MJ. Total energy intake: implications for epidemiologic analyses Am J Epidemiol 1986; 124: 17-27.

23. Cherbuin N, Anstey KJ. The mediterranean diet is not related to cognitive change in a large prospective investigation: the path Through Life Study. Am J Geriatr Psychiatry 2011; Sep: 20

24. Scarmeas N, Stern Y, Mayeux R, Manly JJ, Schupf N, Luchsinger JA. Mediterranean die and mild cognitive impairment. Arch Neurol 2009; 66: 216-225.

25. Roberts RO, Geda YE, Cerhan JR, Knopman DS, Cha RH, Christianson TJ et al. Vegetables, unsaturated fats, moderate alcohol intake, and mild cognitive impairment. Dement Geriatr Cogn Disord 2010; 29: 413-423.

26. Breteler MM. Vascular risk factors for Alzheimer's disease: an epidemiologic perspective. Neurobiol Aging 2000; 21: 153-160.

27. Luchsinger JA, Mayeux R. Cardiovascular risk factors and Alzheimer's disease. Curr Atheroscler Rep 2004; 6: 261-266

28. Cummings JL. Alzheimer's disease. N Engl J Med 2004; 351: 56-67.

29. Scarmeas N, Stern Y, Mayeux R, Luchsinger JA. Mediterranean diet, Alzheimer disease, and vascular mediation. Arch Neurol 2006; 63: 1709-1717.

30. Martins RN, Harper CG, Stokes GB, Masters CL. Increased cerebral glucose-6-phosphate dehydrogenase activity in Alzheimer's disease may reflect oxidative stress. J Neurochem 1986; 46: 1042-1045.

31. Fito M, Cladellas M, de la Torre R, Marti J, Alcantara M, Pujadas-Bastardes M et al. Antioxidant effect of virgin olive oil in patients with stable coronary heart disease: a randomized, crossover, controlled, clinical trial. Atherosclerosis 2005; 181: 149-158.

32. Szeto YT, Tomlinson B, Benzie IF. Total antioxidant and ascorbic acid content of fresh fruits and vegetables: implications for dietary planning and food preservation. Br J Nutr 2002; 87: 55-59.

33. Joshipura KJ, Hu FB, Manson JE, Stampfer MJ, Rimm EB, Speizer FE et al. The effect of fruit and vegetable intake on risk for coronary heart disease. Ann Intern Med 2001; 134: 1106-1114.

34. Witztum JL. The oxidation hypothesis of atherosclerosis. Lancet 1994; 344: 793-795.

35. Byers T, Perry G. Dietary carotenes, vitamin $\mathrm{C}$, and vitamin $\mathrm{E}$ as protective antioxidants in human cancers. Annu Rev Nutr 1992; 12: 139-159.

36. Owen RW, Haubner R, Wurtele G, Hull E, Spiegelhalder B, Bartsch H. Olives and olive oil in cancer prevention. Eur J Cancer Prev 2004; 13: 319-326.

37. Stupans I, Kirlich A, Tuck KL, Hayball PJ. Comparison of radical scavenging effect, inhibition of microsomal oxygen free radical generation, and serum lipoprotein oxidation of several natural antioxidants. J Agric Food Chem 2002; 50: 2464-2469.

38. Mancini M, Parfitt VJ, Rubba P. Antioxidants in the Mediterranean diet. Can J Cardiol 1995; 11(Suppl G): 105G-109GG.

39. Paschos GK, Rallidis LS, Liakos GK, Panagiotakos D, Anastasiadis G, Votteas V et al. Background diet influences the anti-inflammatory effect of alpha-linolenic acid in dyslipidaemic subjects. Br J Nutr 2004; 92: 649-655

40. Fung TT, McCullough ML, Newby PK, Manson JE, Meigs JB, Rifai N et al. Diet-quality scores and plasma concentrations of markers of inflammation and endothelial dysfunction. Am J Clin Nutr 2005; 82: 163-173.

41. Blum S, Aviram M, Ben-Amotz A, Levy Y. Effect of a Mediterranean meal on postprandia carotenoids, paraoxonase activity and C-reactive protein levels. Ann Nutr Metab 2006; 50 : 20-24

42. Steptoe A, Pollard TM, Wardle J. Development of a measure of the motives underlying the selection of food: the food choice questionnaire. Appetite 1995; 25: 267-284.

43. Green J, Waters E, Haikerwal A, O'Neill C, Raman S, Booth ML et al. Social, cultural and environmental influences on child activity and eating in Australian migrant communities. Child Care Health Dev 2003; 29: 441-448.

44. Paulus D, Saint-Remy A, Jeanjean M. Dietary habits during adolescence-results of the Belgian Adolux Study. Eur J Clin Nutr 2001; 55: 130-136.

45. Stafleu A, Van Staveren WA, de Graaf C, Burema J, Hautvast JG. Family resemblance in energy, fat, and cholesterol intake: a study among three generations of women. Prev Med 1994: 23: 474-480.

46. Cunningham SARJ, Venkat Narayan KM. Health of foreign-born people in the United States: a review. Health Place 2008; 14: 623-635.

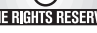

Translational Psychiatry is an open-access journal published by Nature Publishing Group. This work is licensed under the Creative Commons Attribution-NonCommercial-No Derivative Works 3.0 Unported License. To view a copy of this license, visit http://creativecommons.org/licenses/by-nc-nd/3.0/

\section{Supplementary Information accompanies the paper on the Translational Psychiatry website (http://www.nature.com/tp)}

